\title{
Clinical efficacy and safety of transcatheter embolization for vascular complications after percutaneous nephrolithotomy
}

\author{
Necdet Poyraz ${ }^{1}$, Mehmet Balasar ${ }^{1}$, Ibrahim Erdem Gökmen ${ }^{1}$, Osman Koç ${ }^{1}$, Mehmet Giray Sönmez $^{2}$, Arif Aydın², \\ Yunus Emre Göger², Ahmet Öztürk ${ }^{2}$ \\ ${ }^{1}$ Department of Radiology, Necmettin Erbakan University, Meram Medical Faculty, Konya, Turkey \\ ${ }^{2}$ Department of Urology, Necmettin Erbakan University, Meram Medical Faculty, Konya, Turkey
}

Videosurgery Miniinv 2017; 12 (4): 403-408

DOI: https://doi.org/10.5114/wiitm.2017.69108

\begin{abstract}
Introduction: Percutaneous nephrolithotomy (PNL) is the preferred procedure for safe and effective surgical treatment of kidney stones. Hemorrhage is the most serious complication of PNL, resulting from pseudoaneurysm (PA) or arteriovenous fistula (AVF), and can usually be controlled with conservative treatment.

Aim: To evaluate endovascular treatments and outcomes of vascular complications observed after PNL.

Material and methods: We retrospectively reviewed data on 19 patients who underwent renal embolization due to post-PNL renal artery bleeding between March 2005 and September 2016. Embolization materials included embolization coils and glue. The incidence of post-PNL vascular complications and their endovascular treatments, outcomes, and the follow-up data were analyzed.

Results: Nineteen (1.1\%) of 1,609 patients (mean age: 44.9 years, range: 19-75 years) underwent angiography and subsequent transcatheter embolization to control bleeding. The mean time to onset of hemorrhage was 7.2 days after PNL (range: 3-18 days). The PNL entry site was the lower calyx in 15 patients, the middle calyx in 3, and the upper calyx in 1. PA, AVF, and PA plus AVF occurred in 14, 5, and 3 of the 19 renal angiography patients, respectively. Embolization of the affected vessels was successful in all 19 patients. The embolization materials of coil, glue, and coil plus glue were used in 16, 3, and 2 patients, respectively.

Conclusions: Severe hematuria is a rare complication of PNL and can be successfully treated with transcatheter embolization.
\end{abstract}

Key words: percutaneous nephrolithotomy, renal vascular complications, transcatheter embolization, pseudoaneurysm, arteriovenous fistula.

\section{Introduction}

Percutaneous nephrolithotomy (PNL) is the preferred procedure for safe and effective surgical treatment of kidney stones [1]. Hemorrhage is the most serious complication of PNL, resulting from pseudoaneurysm (PA) or arteriovenous fistula (AVF), and can usually be controlled with conservative treatment [2-4]. After PNL, life-threatening hemorrhage requiring transfusion at a rate of $11.2-17.5 \%$ has been reported in the literature [5]. Transarterial embolization (TAE) should be performed in emergency cases with ongoing life-threatening hemorrhages. Fortunately, TAE is required in less than $1 \%$ of patients after PNL [3]. Several studies in the literature describe the endovascular treatment of vascular complications of PNL [6-10]. 


\section{Aim}

We evaluated the incidence of post-PNL vascular complications and their endovascular treatments and outcomes.

\section{Material and methods}

Between March 2005 and September 2016, a total of 1,609 patients underwent PNL at our institution for removal of kidney stones. We retrospectively analyzed the records of 19 patients (12 males, 7 females; age range: $19-75$ years, mean age: 44.9 years) who developed hemorrhage in the postoperative period (range: 3-18 days post-PNL; mean: 7.2 days post-PNL). The PNL entry site was the lower calyx in 15 patients, the middle calyx in 3, and the upper calyx in 1 . Written informed consent was obtained from each patient before angiographic embolization. No approval by the institutional review board was required for this retrospective study.

The 19 (1.1\%) of the 1,609 patients had macroscopic and sometimes massive post-PNL hematuria requiring angiography and embolization for bleeding control, with hematocrit levels decreasing to an average value of $24 \%$ (range: $22-28 \%$ ). The mean hemoglobin reduction was $2.7 \mathrm{mg} / \mathrm{dl}$ (range: 2.0$4.7 \mathrm{mg} / \mathrm{dl}$ ) and all of the patients required blood transfusions, with a mean blood transfusion amount of $2.36 \pm 0.61$ units (range: $2-4$ units).

Serum hemoglobin of $<10 \mathrm{~g} / \mathrm{dl}$, tachycardia associated with gross hematuria (> 100 beats/ min), and hypotension (<90 $\mathrm{mm} \mathrm{Hg}$ ) resulting in hemodynamic instability and requiring blood transfusions were defined as the criteria for post-PNL renal angiography. Sonography and/or computed tomography (CT) were performed in 15 of the patients before angiography. In 9 patients, perirenal hematoma was detected. Four patients underwent immediate angiography without any diagnostic examination. Serum urea and creatinine levels and the glomerular filtration rate (GFR) [11] were analyzed, and pre- and postembolization values were compared to assess the effect of embolization on renal function. Arterial lesions seen on the angiogram were classified into two groups: PA and AVF. The size and localization of the PA according to angiography, the presence of an accompanying AVF, the type of embolic material, the volume of used contrast media, and the length of follow-up were recorded.

\section{Embolization procedure}

After administration of local anesthetic (lidocaine), transfemoral arteriography was performed. The right common femoral artery was entered using the Seldinger technique and a $5 \mathrm{~F}$ sheath was placed. Selective catheterization and angiography (4F or $5 \mathrm{~F}$ Cobra or Simmons 2) of the renal artery was performed. A superselective microcatheter system (Progreat Microcatheter; Terumo Medical) was used for the embolization of the interlobar arteries. The microcatheter was placed through the renal artery in an attempt to approach the lesion as closely as possible, and an embolizing agent was used for occlusion. Embolizing agents consisted of coils (Hilal embolization microcoil and Azur pushable hydrocoil, Terumo) and glue (cyanoacrylate). The embolizing materials were selected during the procedure according to the lesion diameter and the type of hemorrhage. Control angiography was performed after the procedure for the confirmation of total occlusion, then the catheters were pulled and the procedure was terminated. The median volume of contrast media used during angiography was $60 \mathrm{ml}$ (range: 50-80 ml).

After the embolization procedure, each patient underwent follow-up to evaluate the treatment outcomes, complications, and possible recurrent bleeding in the short term and after discharge. The follow-up included clinical and laboratory findings, as well as ultrasonography or CT evaluations when necessary.

\section{Statistical analysis}

All analyses were performed using the Statistical Package for Social Sciences (SPSS) software version 20.0 (IBM SPSS, Inc., Chicago, IL, USA). Continuous variables were expressed as mean \pm standard deviation (and median (range) when indicated). Categorical variables were expressed as frequencies or percentages. Statistical analyses were performed with the paired $t$-test. A $p$-value of $<0.05$ was considered statistically significant.

\section{Results}

All 19 patients underwent angiography to identify the vascular pathology of the bleeding and for treatment with embolization. Among the 19 patients, we identified 14 with PA and 5 with AVF. A combination of PA and AVF was found in 3 patients. The mean PA diameter was $16.8 \mathrm{~mm}$ (range: 4-29 mm). 
The PA was located in the upper pole of the kidney in 2 patients, in the middle pole in 5 , and in the lower pole in 7 , while 12 of the lesions were found in the right kidney and 7 in the left kidney.

In all 19 patients, PAs and AVFs were visualized by superselective catheterization of the renal artery followed by embolization with coil and/or glue. Control angiography was performed to confirm non-filling of the fistula and PA and the success of complete embolization (Photos 1-3). Coil embolization was performed in the 14 PA-only patients and a median of three coils (range: 1-8) were used for each. As embolization material, glue was used in 3 patients and coil plus glue was used in 2 .

The mean duration of follow-up was 17 (range: 1-62) months. Two patients had re-bleeding in the early follow-up period and underwent re-embolization with coils. None of the patients experienced
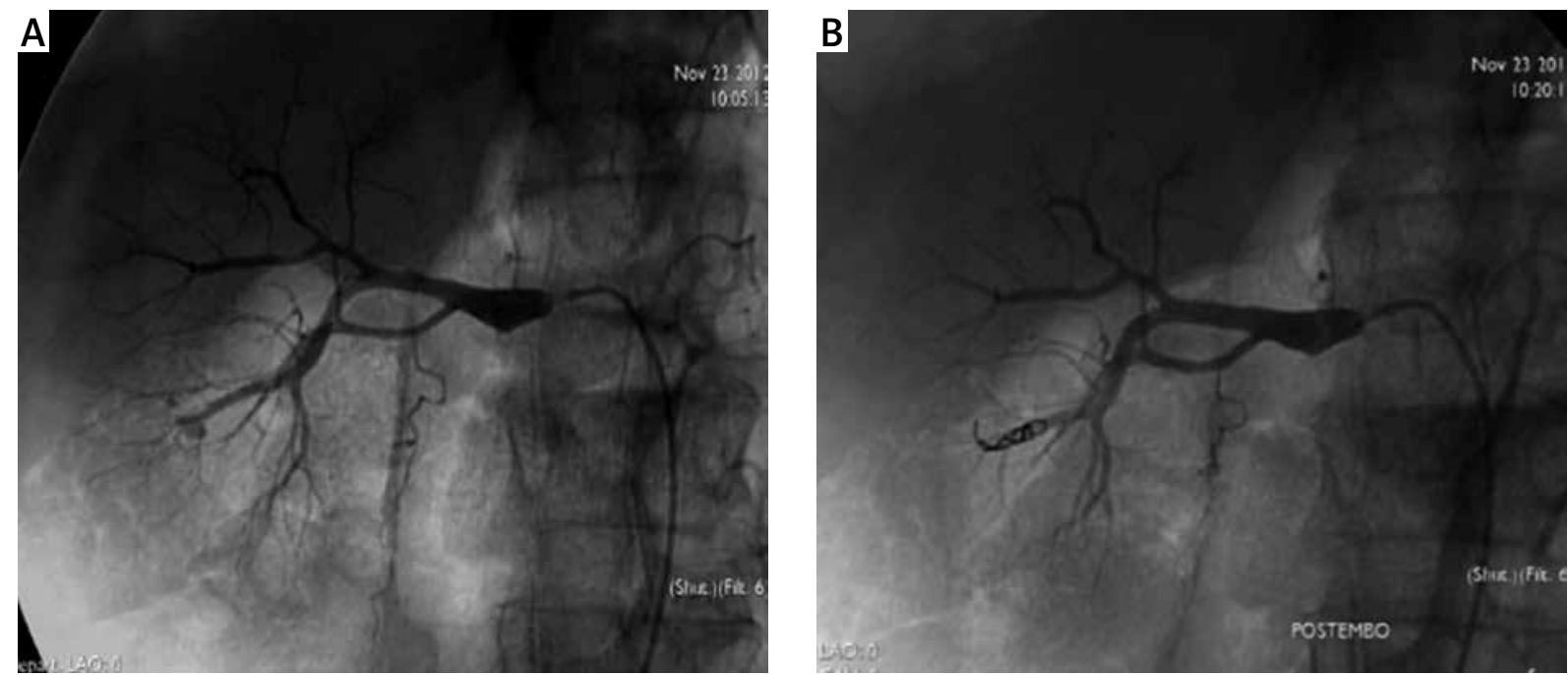

Photo 1. Angiographic confirmation of renal artery pseudoaneurysm (A) and result after embolization using microcoils (B)
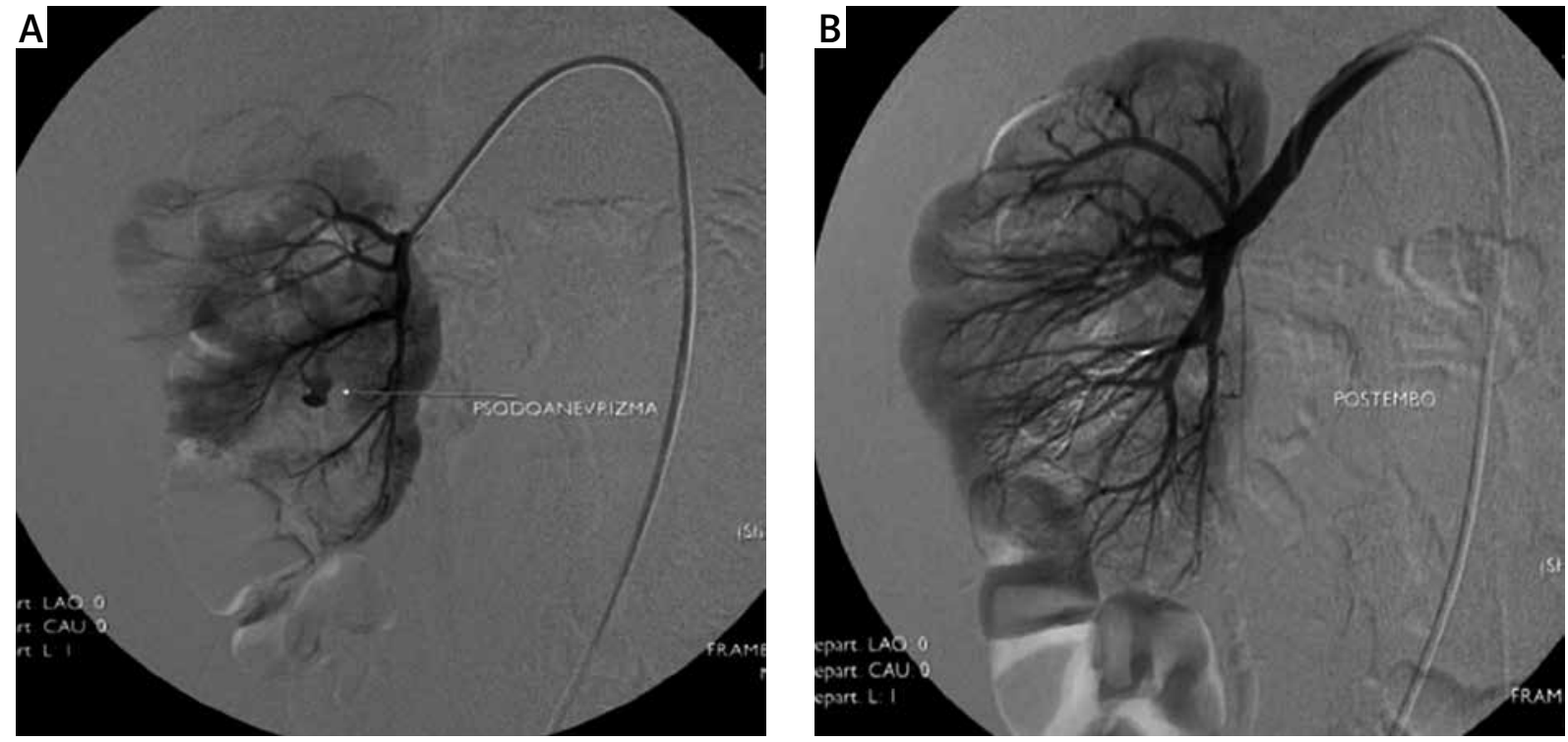

Photo 2. Right superselective renal angiography of a 32-year-old woman who presented with bleeding after PNL. Digital subtraction angiogram showed a pseudoaneurysm (arrow) arising from a lower segmental renal artery branch (A). Control angiogram after glue embolization showing complete obliteration of the pseudoaneurysm (B) 

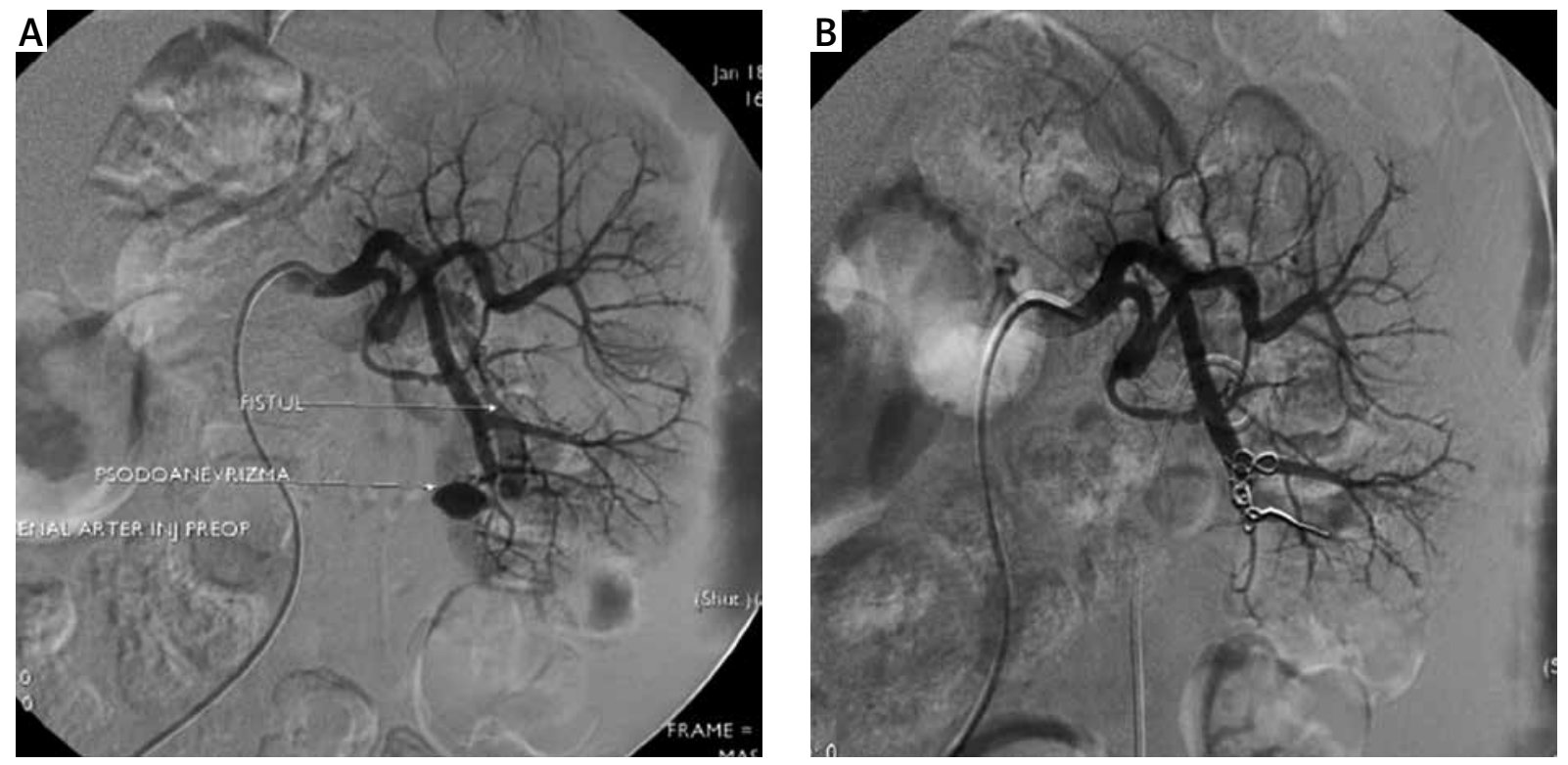

Photo 3. A 75-year-old man with severe hematuria after PNL. Left renal angiography showed a pseudoaneurysm with an arteriovenous fistula (A). Control angiogram after coil embolization showing disappearance of the pseudoaneurysm and arteriovenous fistula (B)

postembolization syndrome, abscess formation, persistent serum creatinine elevation, or hypertension during the procedure and/or follow-up period. Pre- and postembolization values for GFR, serum urea, and serum creatinine levels were not significantly different (Table I). Follow-up ultrasonography showed that after transcatheter embolization (TCE), perirenal hematomas became smaller and finally disappeared completely in all 19 patients.

\section{Discussion}

The PNL has become a common procedure performed on patients with renal calculi, and hemorrhage is the most frequent complication. Excessive bleeding, one of the most important problems observed after PNL, may be observed during the pro-

Table I. Changes in glomerular filtration rate (GFR) and serum parameters

\begin{tabular}{|lccc|}
\hline Parameter & $\begin{array}{c}\text { Before } \\
\text { embolization } \\
(n=23)\end{array}$ & $\begin{array}{c}\text { After } \\
\text { embolization } \\
(n=23)\end{array}$ & $P$-value \\
\hline GFR $[\mathrm{ml} / \mathrm{min}]$ & $71.3 \pm 32.7$ & $65.5 \pm 34.1$ & 0.194 \\
\hline Serum urea $[\mathrm{mg} / \mathrm{dl}]$ & $48.2 \pm 30.3$ & $49 \pm 30$ & 0.455 \\
\hline $\begin{array}{l}\text { Serum creatinine } \\
{[\mathrm{mg} / \mathrm{dl}]}\end{array}$ & $1.6 \pm 1.4$ & $1.7 \pm 1.5$ & 0.202 \\
\hline
\end{tabular}

cedure or in the postoperative period as a result of renal puncture, Amplatz tract dilatation, or renovascular damage caused by difficult manipulation of the nephroscope for especially large calculi. During the procedure, the arterial system is under increased pressure, which means that the risk of an AVF (blood traveling from the injured artery to the adjacent injured vein) or a pseudoaneurysm (blood traveling to the parenchyma) is high [5, 12-14]. These life-threatening hemorrhages are usually caused by PA and AVF, as in our cases.

This study evaluated renal artery lesions that developed after PNL, the endovascular embolization treatments used for them, and the clinical outcomes. All 19 of the included patients were successfully embolized and the bleeding was stopped. Only 2 patients developed re-bleeding in the early period, which was treated by re-embolization. Postembolization syndrome and deterioration of renal function were not observed on follow-up after TCE.

The PA is a hematoma surrounded by fibrous tissue or the adventitia of the artery that occurs as a result of arterial leakage; the blood within the hematoma relates to the artery through an aperture. With these structural characteristics, PAs have high clinical importance due to the high risk of rupture. Rupture risk seems to be higher in PAs compared to real aneurysms. Renal artery PAs may be asymptom- 
atic; however, when the size of the aneurysm reaches twice the normal vessel size, the risk of rupture increases and embolization becomes necessary. All PAs should be immediately treated once they are detected at any size. In the past, surgical exploration or nephrectomy was performed for such situations, but these operations carry a high mortality rate of 30-50\% [14-16].

Recent developments in interventional radiological techniques have made superselective catheterizations possible [17-19], and PAs may now be treated with minimally invasive procedures using embolization materials. Embolization coils may be used alone or in combination with other non-resorbable materials, such as glue or onyx. These materials can effectively maintain kidney function while providing rapid hemostasis [5, 20, 21].

The AVF is an abnormal communication between an artery and a vein without involving a capillary bed, and may be asymptomatic or cause macroscopic hematuria. The concomitance of PA and AVF is not yet clearly understood $[3,9]$. In our study, PA and AVF were present together in 3 of 19 cases. The AVF is frequently observed after percutaneous surgical interventions, after partial nephrectomy, or post-traumatically. Surgical treatment includes vascular ligation with partial or total nephrectomy. Selective embolization techniques are increasingly preferred for AVF since they maintain the renal parenchyma and reduce morbidity rates [6, 20-22].

Richstone et al. [23] reported that TCE was required in 57 (1.2\%) of 4,695 patients following percutaneous renal interventions (PNL was performed in 44 of the 57 patients). Srivastava et al. [3] reported embolization in 24 (1.2\%) of 1,854 patients following PNL, with combined PA and AVF in 4 patients. Martin et al. [16] reported severe bleeding in 8 of 808 patients following PNL, 7 of whom were treated with TCE. In our series, 19 (1.1\%) of 1,609 patients underwent TCE following PNL. This $1.1 \%$ rate is in accordance with the literature.

Selection of embolizing agents for endovascular embolization therapy is performed based on PA and AVF localization on angiography, as well as on tortuosity of the vessels, width and flow rate of the AVF, and width of the neck of the PA.

The complication rate for the TCE procedure is relatively low. The most important complication reported in the literature is vascular obstruction due to reflux of the embolizing agent from the target and related minimal infarctions that do not impair renal function [6, 21, 22]. Ischemic complications due to reflux of the embolizing material from the target in liquid embolizing agents is relatively low with coil usage [17-19]. Usage of liquid embolizing agents, such as glue, is more difficult compared to the coil material and requires experienced operators. It is important to push the microcatheter forward just until it is proximal to the area to be embolized, and to then begin the procedure to prevent reflux of the embolization material. Successful outcomes are obtained from embolization procedures performed by experienced operators and when the highest level of tissue protection is provided [4, 20, 21]. In our study, no reflux of coil material to different locations was observed.

The CT is highly useful in the diagnosis of renal parenchymal and pyelocalyceal injury, main renal arterial occlusion, and perirenal hematoma. However, it is not accurate in diagnosing branch arterial injuries, such as PAs or AVFs [24]. Indeed, in our 19 cases, we were able to detect perirenal hematoma only with $\mathrm{CT}$.

Some limitations of our study were that it was retrospective and had a small sample size, with short and variable follow-up periods. Another limitation was that only coil and glue were used, and no other embolizing agents were evaluated. Multi-center studies with larger sample sizes and longer and regular follow-up periods are necessary.

\section{Conclusions}

Severe hematuria is a rare complication of $\mathrm{PNL}$ that can be successfully managed with angioembolization. Based on our case series, this technique has become the first-choice treatment option because of its high success rate, low complication rate, and rapid treatment of severe kidney hemorrhages. Urologists should be in close contact with interventional radiologists to effectively treat serious complications after PNL and to avoid major surgery.

\section{Conflict of interest}

The authors declare no conflict of interest.

\section{References}

1. Skolarikos A, Alivizatos G, de la Rosette JJMCH. Percutaneous nephrolithotomy and its legacy. Eur Urol 2005; 47: 22-8.

2. Le WJ, Smith D, Cubelli V, et al. Complications of percutaneous nephrolithotomy. Am J Roentgenol 1987; 148: 177-80. 
3. Srivastava A, Singh KJ, Suri A, et al. Vascular complications after percutaneous nephrolithotomy: are there any predictive factors? Urology 2005; 66: 38-40.

4. Guneyli S, Gok M, Bozkaya H, et al. Endovascular management of iatrogenic renal arterial lesions and clinical outcomes. Diagnostic Interv Radiol 2015; 21: 229-34.

5. Caliskan KC, Ulusay SM, Tanriverdi O, et al. How and when to perform transcatheter embolization in iatrogenic renal hemorrhage? A new algorithm. Med J Okmeydani Train Res Hosp 2012; 28: 84-93.

6. Zabkowski T, Piasecki P, Zielinski H, et al. Superselective renal artery embolization in the treatment of iatrogenic bleeding into the urinary tract. Med Sci Monit 2015; 21: 333-7.

7. Vijay MK, Vijay P, Das RK, Kundu AK. Renal artery pseudoaneurysm following percutaneous nephrolithotomy. Saudi J Kidney Dis Transpl 2011; 22: 347-8.

8. Massulo-Aguiar MF, Campos CM, Rodrigues-Netto NJ. Intrarenal pseudoaneurysm after percutaneous nephrolithotomy. Angiotomographic assessment and endovascular management. Int Braz J Urol 2006; 32: 440-4.

9. Jinga V, Dorobat B, Youssef S, et al. Transarterial embolization of renal vascular lesions after percutaneous nephrolithotomy. Chirurgia (Bucur) 2013; 108: 521-9.

10. Kervancioglu S, Gelebek Yilmaz F, Erturhan S. Endovascular management of vascular complications after percutaneous nephrolithotomy. Vasa 2014; 43: 459-64.

11. Levey AS, Bosch JP, Lewis JB, et al. A more accurate method to estimate glomerular filtration rate from serum creatinine: a new prediction equation. Ann Intern Med 1999; 130: 461-70.

12. El-Nahas AR, Shokeir AA, El-Assmy AM, et al. Post-percutaneous nephrolithotomy extensive hemorrhage: a study of risk factors. J Urol 2007; 177: 576-9.

13. Gavant ML, Gold RE, Church JC. Delayed rupture of renal pseudoaneurysm: complication of percutaneous nephrostomy. AJR Am J Roentgenol 1982; 138: 948-9.

14. Gremmo E, Ballanger P, Dore B, Aubert J. Hemorrhagic complications during percutaneous nephrolithotomy. Retrospective studies of 772 cases. Prog Urol 1999; 9: 460-3.

15. Lee WJ, Smith AD, Cubelli V, et al. Complications of percutaneous nephrolithotomy. AJR Am J Roentgenol 1987; 148: 177-80.

16. Martin X, Murat FJ, Feitosa LC, et al. Severe bleeding after nephrolithotomy: results of hyperselective embolization. Eur Urol 2000; 37: 136-9.

17. Soyer P, Desgrippes A, Vallee JN, Rymer R. Intrarenal pseudoaneurysm after percutaneous nephrostolithotomy: endovascular treatment with N-butyl-2-cyanoacrylate. Eur Radiol 2000; 10: 1358.

18. Won Y, Lee SL, Kim Y, Ku YM. Clinical efficacy of transcatheter embolization of visceral artery pseudoaneurysms using N-butyl cyanoacrylate (NBCA). Diagn Interv Imaging 2015; 96: 563-9.

19. Calarn P, Nonent M, Fournier G, et al. [Endovascular treatment of traumatic and iatrogenic intrarenal arterial lesions by microcoil embolization]. Ann Radiol 1996; 39: 234-49.

20. el-Nahas AR, Shokeir AA, Mohsen T, et al. Functional and morphological effects of postpercutaneous nephrolithotomy superselective renal angiographic embolization. Urology 2008; 71: 408-12.
21. Poulakis V, Ferakis N, Becht E, et al. Treatment of renal-vascular injury by transcatheter embolization: immediate and longterm effects on renal function. J Endourol 2006; 20: 405-9.

22. Schwartz MJ, Smith EB, Trost DW, Vaughan EDJ. Renal artery embolization: clinical indications and experience from over 100 cases. BJU Int 2007; 99: 881-6.

23. Richstone L, Reggio E, Ost MC, et al. Hemorrhage following percutaneous renal surgery: characterization of angiographic findings. J Endourol 2008; 22: 1129-35.

24. Chen X, Borsa JJ, Dubinsky T, Fontaine AB. CT of a renal artery pseudoaneurysm caused by a stab wound. Am J Roentgenol 2002; 178: 736.

Received: 30.03.2017, accepted: 3.07.2017. 